\title{
Spatial and temporal analysis of the seasonal and interannual variability in the tropical Pacific simulated with a coupled GCM
}

\author{
Francisco J. Álvarez García, William D. Cabos Narváez and María J. Ortiz Beviá \\ Departamento de Física, Facultad de Ciencias, Universidad de Alcalá, Madrid, Spain
}

\begin{abstract}
In the first part of this work, the dominant time scales that explain the tropical variability of the first SINTEX simulation (ECHAM4(T30)-ORCA) are identified through a spectral analysis. Higher order spectral analysis is used to examine the interactions among these time scales. The time series analyzed are an average of sea surface temperature over the Niño3 region. The time scales obtained are compared with those identified in another coupled GCM simulation (ECHAM4(T42)-OPYC3). The higher importance of the biannual time scale in this last is explained partly by the strength of the coupling between the annual and the biannual time scales. There is no such strong coupling in the SINTEX simulation. Important differences among the generation of the simulated warm (or cold) event suggest the need of a systematic classification to isolate their relevant features. Therefore in the second part of this work, we address this problem. A space-time cluster analysis is performed on a data set built by collecting the values of the heat content anomalies in the tropical Pacific region, in the fifteen months previous to a peak in the Niño3 Index that has been identified as a 'warm' (or 'cold') event. In the case of the warm events, three types of generation schemes are found. In two of them, there are anomalies of heat content in the west, north and south of the equator, more than nine months before the events start. In the third case, the anomalies appear and grow in the central equatorial Pacific. Only two types are needed to classify the generation of cold events. Negative sea level height anomalies appear six months before the Niño3 Index reaches the (local) minimum. They are located north of the equator in one of the groups, and south of it in the other. Some of these characteristic traits also appear in observations of warm and cold events.
\end{abstract}

Key words coupled model - El Niño-Southern Oscillation - seasonal - interannual - heat contentclusters

\section{Introduction}

In the present paper, we use different statistical techniques to study the tropical Pacific variability found in the ECHAM4(T30)-ORCA

Mailing address: Mr. Francisco J. Álvarez García, Departamento de Física, Facultad de Ciencias, Universidad de Alcalá, Alcalá de Henares, Madrid 28871, Spain; e-mail: franciscoj.alvarez@uah.es simulation for SINTEX. The most usual tool for the monitoring of this region is the Niño3 Index. Built as an average of the anomalies of Sea Surface Temperature (hereinafter SST) within the area $\left(150^{\circ} \mathrm{W}-90^{\circ} \mathrm{W}, 5^{\circ} \mathrm{S}-5^{\circ} \mathrm{N}\right)$, it provides a time series from which the warm and cold events characteristic of the interannual variability can be readily identified (Trenberth, 1997). An equivalent index is employed here for the SST, rather than for its anomalies, produced by two coupled model experiments, the above mentioned SINTEX run and another one performed with ECHAM4(T42)-OPYC3. The dominant time scales of variability in these series are 
determined by means of a standard (second order) spectral analysis.

In the eastern equatorial Pacific, atmospheric processes are known to be not yet correctly represented in General Circulation Models (GCM). Therefore, differences in the location and strength of the spectral peaks between the simulation and observations are not unexpected. On the contrary, the presence in the SINTEX simulation of some realistic spectral features (a peak with a period of four years) that do not appear in the ECHAM4OPYC analysis is interesting. It cannot be related to a better performance in the simulation of the mean state, which is closer to the observed one in the case of ECHAM4-OPYC. A possible explanation could be the existence of more realistic interactions among the different timescales in the SINTEX simulation. In order to test this point, we have resorted to the help of higher order spectral analysis.

There are similarities in the way in which observed warm events start and evolve, and also often in the stage previous to the onset, allowing the identification of a common generation scheme. This is best captured in terms of Sea Level Height (SLH) anomalies. Well before the onset, SLH anomalies appear in the West Pacific, north and south of the equator (Wyrtki, 1985), onto which they converge some time afterwards, propagating eastward and setting on the event. But exceptions to this rule are not lacking. For instance, things happened differently in the case of the 1991-1993 warm event. Thanks to the better sampling, in space and time, provided by coupled simulations, these differences can be further investigated.

For their study of the generation of the warm and cold events in the equatorial Atlantic simulated in the coupled ECHAM4-OPYC3 run, Cabos Narváez et al. (2002) have devised a statistical procedure that seems to perform satisfactorily. It has also been applied with a similar purpose to the study of the same simulation's ENSO events (Álvarez García et al., 2002). The same procedure will be used here to study the ENSO generation in the SINTEX simulation with ECHAM4(T30)-ORCA. The warm and cold events in the latter run have been identified from the Niño3 Index and grouped into distinct classes by means of a spatio-temporal analysis on the values of the anomalies of Heat Content (hereinafter HC), the coupled GCM proxy for SLH anomalies, during the period previous to the peak of each event. 100 years of SINTEX simulation have been analyzed and three (two) groups found for the warm (cold) events.

In Section 2 of this paper, we give details about the simulated data under study. The spectral analysis of the simulated Niño3 time series is presented in Section 3. The methodology followed to sort out the warm ENSO events is explained in Section 4, together with the results obtained. Those corresponding to the classification of the cold events are given in Section 5. Section 6 examines connections with climatic signals external to the tropical Pacific. We finish with a section where methods and results are summarized and discussed.

\section{Data}

The data used for this study were basically those extracted from the first experiment carried out for SINTEX: the ECHAM4 atmospheric model (Roeckner et al., 1996) at T30 resolution coupled to the ORCA oceanic model (Madec et al., 1998). Although differences between the observed and simulated mean state are still important in some regions (for instance the eastern equatorial Pacific), the modelled interannual variability seems to be quite realistic (Gualdi et al., 2003). No trend either in surface or subsurface variables was detected in the 100 year chunk selected for the analysis, that corresponds to years 101 through 200 of the simulation. The spatial domain selected goes from $110^{\circ} \mathrm{E}$ to $70^{\circ} \mathrm{W}$ and from $15^{\circ} \mathrm{S}$ to $15^{\circ} \mathrm{N}$. The grid resolution in the domain is 2 degrees in longitude and varies from 0.8 degrees at $15^{\circ} \mathrm{N}$ and $15^{\circ} \mathrm{S}$ to 0.5 degrees at the equator in latitude.

Anomalies were computed by subtracting this period's climatology from the monthly values of the fields of interest, which are mainly two: the SST and the HC. The latter is the thermal energy stored in the upper oceanic layers, and will be taken here as the model's proxy for the sea level height. It is an optimal variable to monitor the event's generation (Chao and Philander, 1993). 
Let it be defined by

$$
H C\left(t, \vec{r}_{H}\right)=\int_{h_{0}}^{0} \rho c_{p} T\left(t, \vec{r}_{H}, \partial\right) d z
$$

the depth $h_{0}$ taking the value of $300 \mathrm{~m}$. For the sake of the discussion, other simulated variables like the wind stress will also be used.

Additionally, we will analyze an SST Index obtained from data of an ECHAM4-OPYC3 coupled model run (Roeckner et al., 1995 or Bacher et al., 1996). The atmospheric component has a T42 resolution, while the oceanic component has a comparable zonal resolution, but a much coarser vertical discretization (only eleven layers). In a recent intercomparison study of 16 models (Achuta Rao et al., 2000), this CGCM simulation was rated among the best by its simulated seasonal cycle, the statistical characteristics of several climatic indices and the SOI-Niño3 teleconnections. Some of the deficiencies of the model, common to most of them, are warmer than observed SST anomalies in the tropical Pacific off the South American coast and the overestimation of the biannual component of ENSO (Bacher et al., 1998).

\section{Spectral characteristics of the simulated ENSO variability}

A preliminary spectral analysis (not shown) was carried out on the SINTEX simulation of the Niño3 Index. In the spectrum obtained, the biennial spectral peak is the dominant feature, and is overestimated with respect to the observations. This is a characteristic that the SINTEX run shares with other coupled model experiments, and particularly with the ECHAM4-OPYC3. Another lower frequency peak, with a four years period, is found in the SINTEX data, that is missing in the ECHAM4-OPYC3. As it happens, such a peak is present in the observed spectrum of the Niño3 Index and stronger than the biennial one there.

Reasons for the spectral differences between the two simulations can be found in the differences between their respective mean states. But, as mentioned above, the mean state of the ECHAM4-OPYC run is more 'realistic' than the one of the SINTEX simulation. To search for other possible clue, we will look for scale interactions in a higher order spectral analysis. Because the interactions of the interannual variability with the seasonal cycle could be of relevance for our study, we will retain the latter in the time series to be analyzed here. For this reason, the spectra represented in fig. 1 were obtained from SST (not anomalies!) in the Niño3 region. The most important spectral diagnostics as regards the comparison of the modelled and observed seasonal cycle is the overestimation of the semiannual signal in the formers, that could be traced back to the failure in the representation of the atmospheric feedbacks in the Eastern Pacific. At the interannual timescale, the most outstanding features are the excess of power at the biennial period in both simulations, and the four years peak, noticeable in the SINTEX spectrum. Its lack of significance is a side effect of the inclusion of the climatological cycle in the spectral analysis.

To study nonlinear interactions among the spectral peaks, higher order spectral functions have to be computed. The one we use here is the squared bicoherence, given by

$$
b^{2}\left(f_{1}, f_{\eta}\right)=\frac{\left|B_{x}\left(f_{1}, f_{2}\right)\right|^{2}}{E\left\{\left|F(f)(F) f_{2}\right|\right\} E\left\{F\left(f_{1}+f_{\eta}\right) F^{*}\left(f_{1}+f_{7}\right)\right\}}
$$

at the pair of frequencies $\left(f_{1}, f_{2}\right)$. $B$ denotes the true bispectrum of the process under study, and $F$ its Fourier transform, while $E\{$.$\} stands for the$ expected value of the magnitude inside the brackets. The terms in the right hand side of the expression are estimated by averaging previous computations obtained for a number of segments into which the time series is split. Bounded between 0 and 1, the final estimate of the squared bicoherence provides a measure of the degree of phase coupling (indicative of a possible nonlinear interaction) between distinct time scales present in the series. Given the distribution of the estimates (Elgar and Sebert, 1989) under the hypothesis of zero bicoherence, i.e. no phase coupling at all, values significantly greater than zero (at a chosen level, here 95\%) can be determined. They are depicted in fig. 1, right, to show which interactions might be present in the indices under study. 

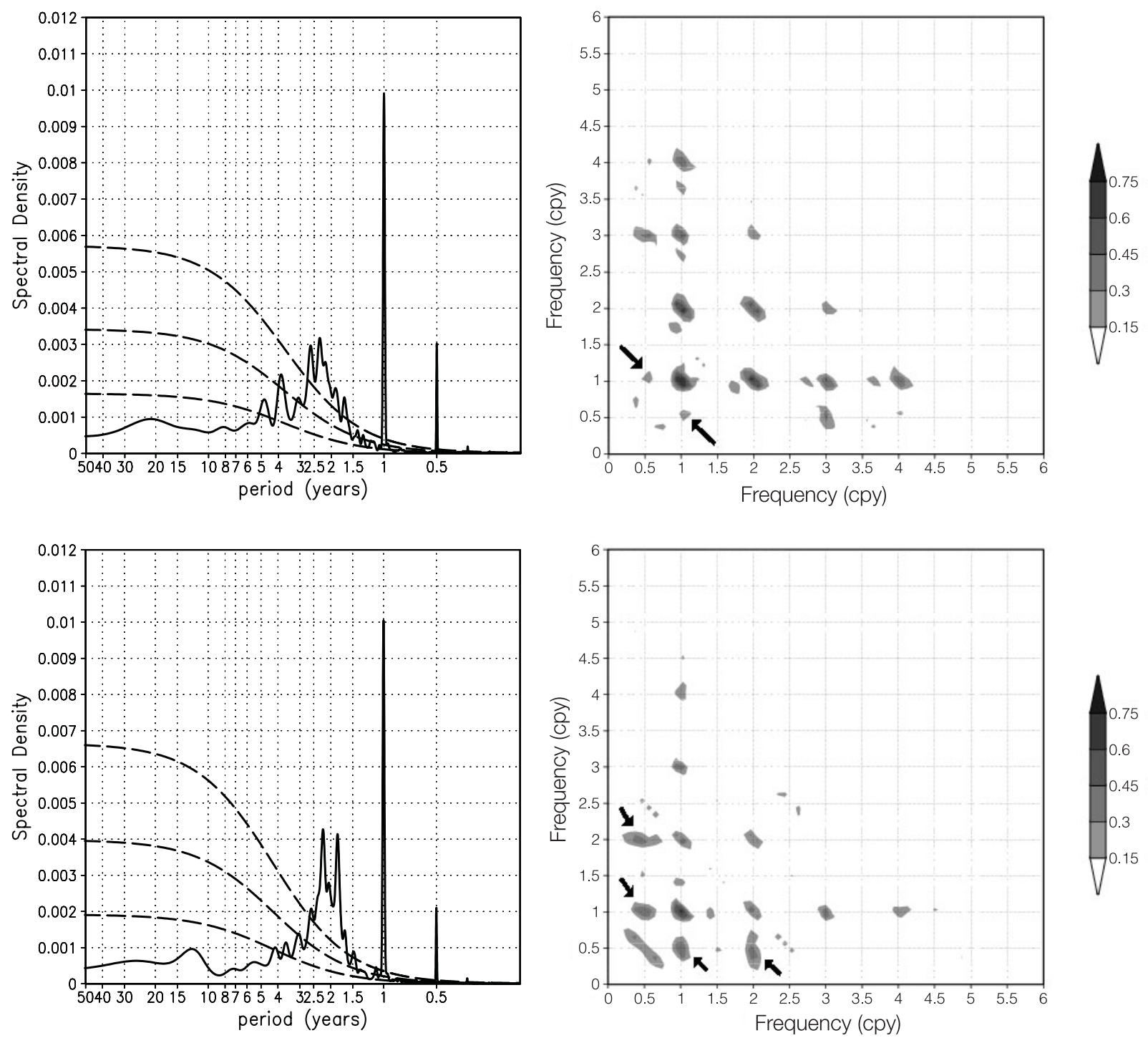

Fig. 1. Power spectrum (left) and bicoherence of the Niño3 SST Index for: (top) SINTEX simulation, (bottom) ECHAM4-OPYC. Spectra were computed using a Parzen lag-window of width 600 months for both simulations.

In the SINTEX simulation, we find strong nonlinear interaction only among the annual and the intra-annual peaks (semiannual, four and three months periods). These interactions, albeit weaker, are also present in the ECHAM4OPYC3 analysis, which in addition shows strong interactions between the two years peak and the annual and semiannual ones (marked with arrows in fig. 1). We hypothesize that this nonlinear interaction, that can be related to the flux correction applied in this run, would dissipate an energy that otherwise would be moved to the lower frequencies.

\section{Classification of warm events according to their generation}

\subsection{Methodology}

In order to identify the warm ENSO events in the sample, the Niño3 Index was used to spot out the periods when anomalies exceed the threshold of one standard deviation of the time series, with the condition that two events cannot occur within the same year (Trenberth, 1997). This criterion identifies correctly the events in the observed Niño3 Index, and yields 34 warm 
events in the 100 years chunk analyzed for the SINTEX run. The highest peak in the Niño3 SST anomaly during the period of occurrence of an event, will be used as a reference in what follows, as detailed below.

Once the events have been identified, we proceed to their classification. For each warm event identified, data covering the Pacific basin within $15^{\circ} \mathrm{S}-15^{\circ} \mathrm{N}$ for the 15 months previous and the month where the Niño3 Index peaks are selected. An ensemble is formed by collecting the 15-month-long chunks corresponding to the different events. To this ensemble, we apply an agglomerative cluster analysis based on the minimization of the following distance between two events

$$
d_{p q}^{l}=+s_{p q}^{l}
$$

with

$$
s_{p q}^{l}=\frac{\sum_{j=1}^{N} y_{p j}(y) y_{q d} l}{\sqrt{\left(\sum_{j=1}^{N} y_{p j}^{2}(\gamma)\right){ }_{j 1}^{N} y_{q j}^{2}(\chi)}}
$$

where $y_{p}(l)$ and $y_{q}(l)$ stand for the spatial patterns of the $\mathrm{HC}$ anomalies in events $p$ and $q$ respectively, $l$ months before their respective peaks in the SST anomaly Niño3 Index. Thus defined, the distance $d_{p q}^{l}$ will vary between 0 and 2 . The delay $l$ is computed with respect to the peak of Niño3 Index in each event. That is, in the following, month -1 , for instance, refers to the month previous to the peak, and month +1 to the one after. Notice that the peak of the Niño3 Index does not correspond to the 'peak phase' of Rasmusson and Carpenter's composites (1982), but rather to an intermediate stage near 'transition phase', as pointed out by Latif et al. (1993).

The classification is carried out in two stages: at a first stage, when clusters are still undefined, values of $d_{p q}^{l}$ for every pair of events, with $l$ ranging between -15 and 0 months, are computed. An average (over $l$ ) of these distances is used to establish the initial groups, which are formed by a few events (typically 4 or 6 ) with minimum distance (under a threshold of 0.3) among them. Each group is then characterized by a composite at each lag $l$ and on the second stage, the distance to these composites is computed for all the events that remain unclassified. Each one is aggregated to its nearest group, using as a criterion the distance averaged over the values of $l$ from -10 to -5 , chosen for the sake of a more distinct classification. The latter's robustness, however, has been examined, with positive results, by using other intervals within the $(-15,0)$ months range.

\subsection{Results}

The results of our classification of the warm events are summarized in table I. The good performance of this classification is evidenced by the fact that the one standard deviation intervals for distances within and outside a given group do not overlap. Most of the 34 events are distributed among three groups, that are characterized by their HC composites at different lags. In the Hoevmueller diagrams of the fig. 2, the evolution in time (lag before and after the peak) of the $\mathrm{HC}$ composite at the equator for each of the three clusters is represented in the top row. In the bottom row, the corresponding SST composites for each type are shown. As can be seen there, in events of type I and II there is an accumulation of anomalies of $\mathrm{HC}$ west of the dateline, fifteen months before the peak of the events, while in events of type III, anomalies appear only eight months before the peak. The accumulation of $\mathrm{HC}$ anomalies in the west for events of types I and II has an extra-equatorial origin; the role of the extra-equatorial regions at this stage is much less relevant in the case of events of type III.

Although events of type I and type II are similar in that regard, there are also differences

Table I. Warm events classification results. N1, D1, S1: number of elements, average distance and standard deviation of the latter within a given group. N2, D2, S2: same as above for events outside a given group. FV: fractional contribution to the variance of the Niño3 Index due to all warm events by those in a given group.

\begin{tabular}{cccccccc}
\hline \hline Group & N1 & D1 & S1 & N2 & D2 & S2 & FV \\
\hline I & 12 & 0.32 & 0.10 & 22 & 0.75 & 0.20 & $32 \%$ \\
II & 9 & 0.20 & 0.09 & 25 & 0.82 & 0.24 & $47 \%$ \\
III & 5 & 0.40 & 0.09 & 29 & 0.70 & 0.16 & $12 \%$ \\
\hline
\end{tabular}



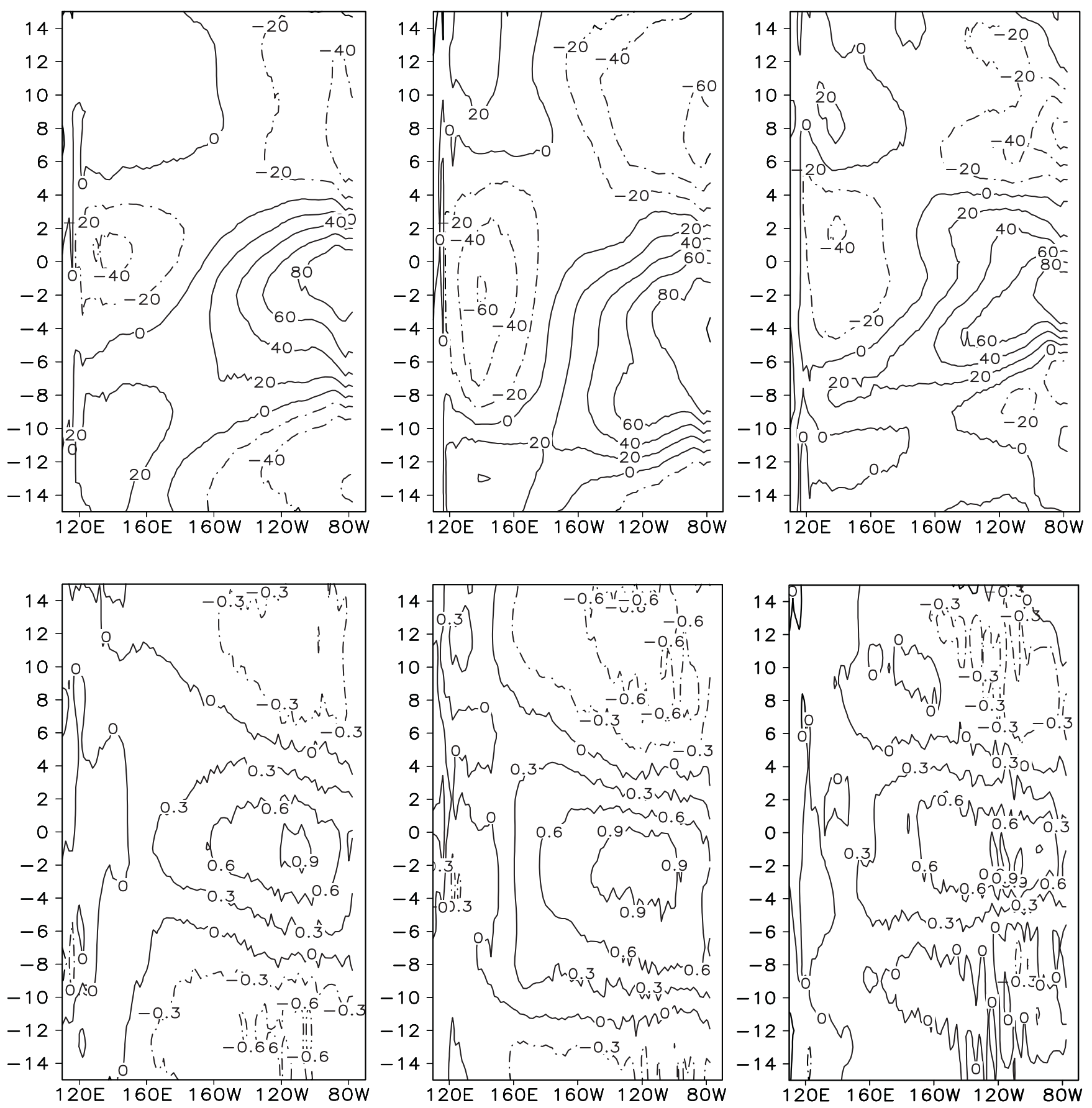

Fig. 2. Hoevmueller diagrams showing the evolution of (top) Heat Content $\left(10^{7} \mathrm{Jm}^{-2}\right)$ and (bottom) SST $\left({ }^{\circ} \mathrm{C}\right)$ anomalies averaged within the equatorial strip $3^{\circ} \mathrm{S}-3^{\circ} \mathrm{N}$ for warm events of type I (left), II (center) and III (right).

between them: events of type II present a greater strength in the characteristic west-east HC gradient, and they start before and last longer (almost one year). More differences can be detected in the extra-equatorial area.

\subsection{The warm events seasonal phase locking}

Phase locking between ENSO and the seasonal cycle has received much attention. For the events included in Rasmusson and Carpenter 
(1982) composites (those from 1950 to 1981), the phase locking was evident, the Niño3 Index peaking in late summer (July-August-September). In recent events (1982-1983 or 1997-1998) the peak of the Niño3 Index occurs later in the year
(Neelin et al., 2000), a feature that has sometimes been connected to climate change.

It is interesting to check if our classification can be related to the seasonal phase. In the fig. 3a we have represented the seasonal signature of

(a)
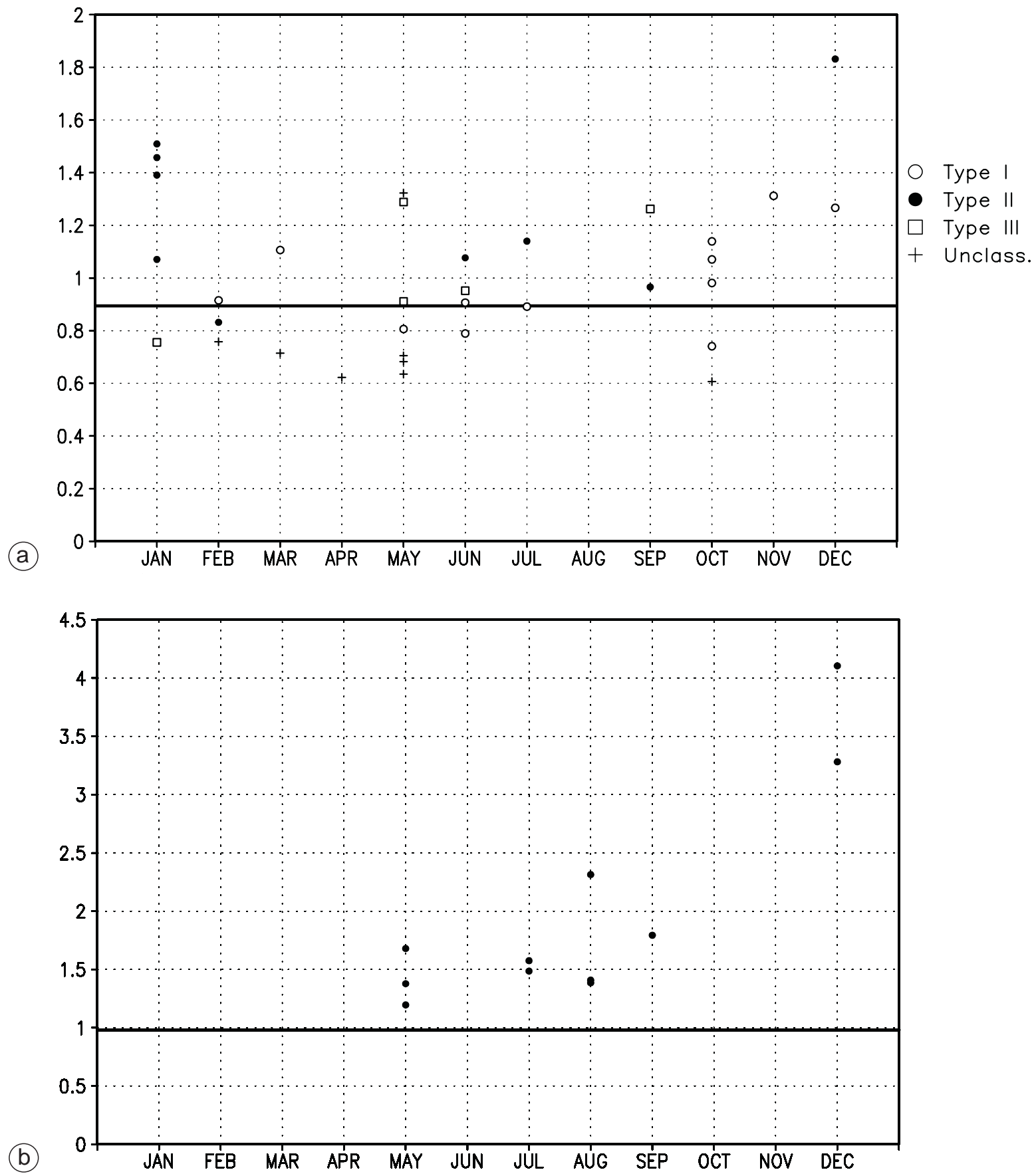

Fig. 3a,b. a) Seasonal distribution of warm SST anomaly peaks in the SINTEX simulation. The horizontal line marks the 1.5 standard deviation threshold for the Niño3 Index; b) as in (a), but for the observed SST anomalies (COADS + IGOSS, 1950-1999) peaks. 

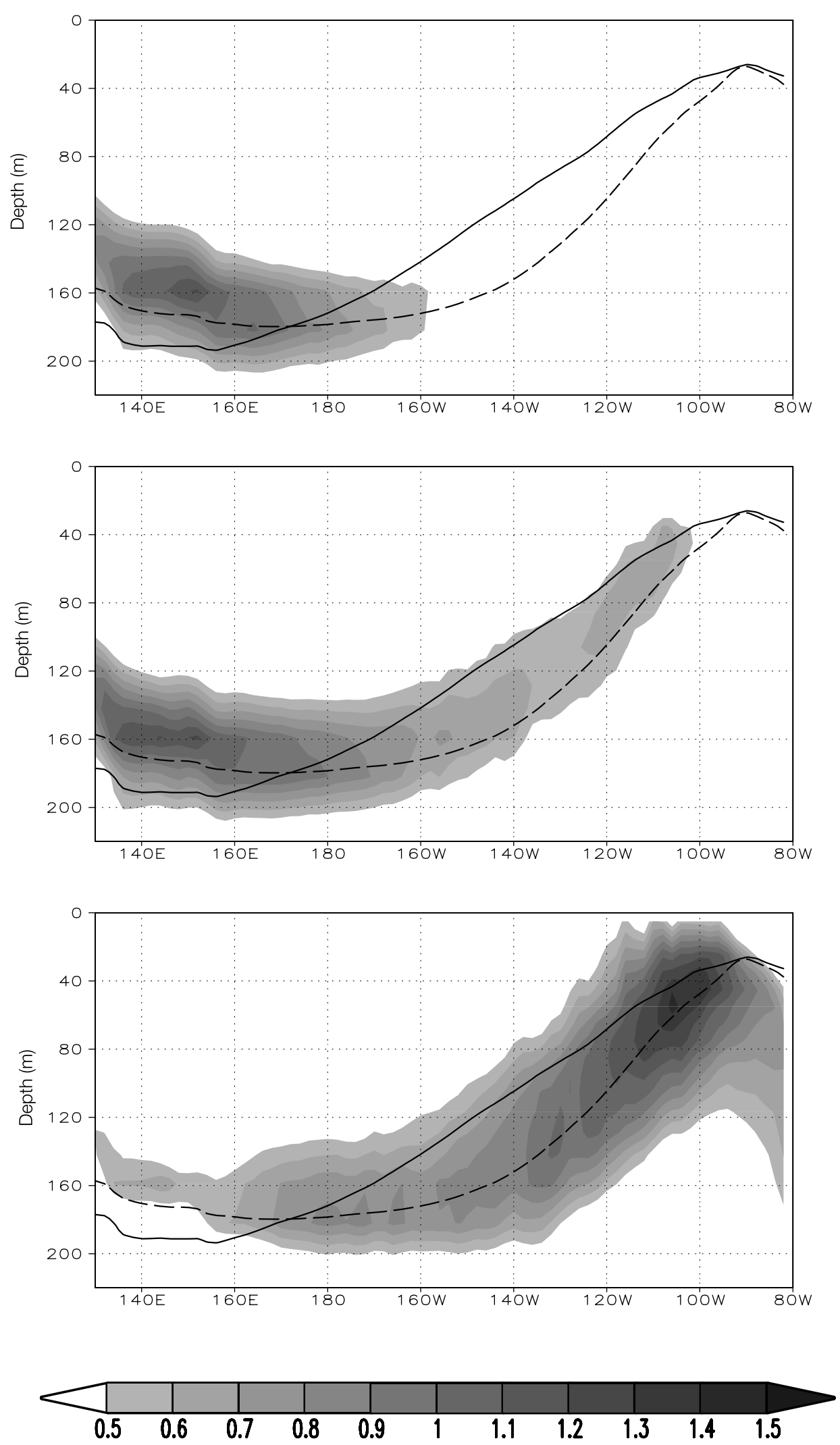

Fig. 4. Vertical section of temperature anomalies $\left({ }^{\circ} \mathrm{C}\right)$ along the equatorial Pacific for warm events of type II in months -13 (top), -11 (middle) and -9 (bottom). Superimposed are the climatological $20^{\circ} \mathrm{C}$ isotherm in January (broken line) and March (solid line). 

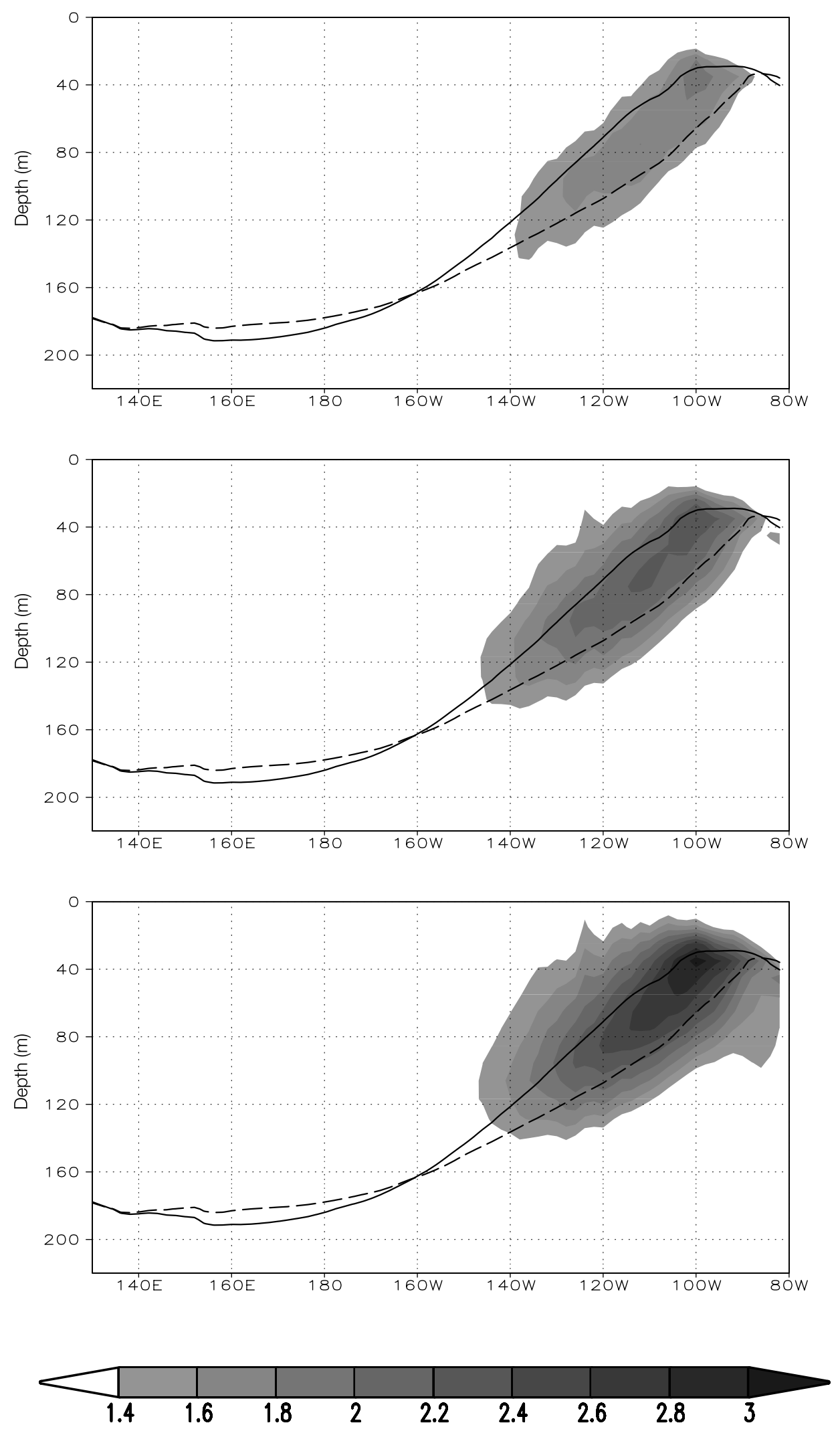

Fig. 5. Vertical section of temperature anomalies $\left({ }^{\circ} \mathrm{C}\right)$ along the equatorial Pacific for warm events of type II in months -6 (top), -5 (middle) and -4 (bottom). Superimposed are the climatological $20^{\circ} \mathrm{C}$ isotherm in July (broken line) and September (solid line). 
the warm events in the SINTEX run, marked by a dot in the month when the Niño3 Index peaks, together with a measure of their amplitude as given by their maximum value in the index. In order to highlight the stronger events, a straight horizontal line corresponding to an anomaly 1.5 times the standard deviation of the index has been drawn. We can appreciate a preference for events of type I to peak more frequently and strongly in autumn, while events of type II, that are among the more intense ones, peak preferentially in winter. Such a tendency might be linked to an interaction with the mean seasonal cycle, as fig. 4 suggests. In the upper panel, we display a vertical section of the anomalies of temperature along the equator in month -13 of the type II events composite; warm subsurface anomalies are evident in the western margin. These anomalies are seen to travel eastward, rising along the shallower thermocline, in the following months, as depicted in the middle (month -11) and lower (month -9 ) panels. For events of type II with Niño3 Index peaking in winter (December-January), the transit just described corresponds to December (month - 13) to April (month -9 ), and coincides with the climatological rise of the thermocline illustrated in fig. 5 by the change in the $20^{\circ} \mathrm{C}$ isotherm from January to March. Later on in the evolution of events type II, during months -6 to -4 , there is an intensification in the subsurface temperature anomalies in the Eastern Pacific, displayed in the three panels in fig. 5, in response to Central Pacific highest positive zonal wind anomalies (not shown). Again for those events of type II with Niño3 Index peaking in winter, the latter enhancement of the warm temperature anomalies occurs in July (month -6) - September (month $-4)$, when the climatological thermocline shallows in the east due to a reinforcement of the trades. The coincidence of these two relevant stages in the development of the events, namely, the passage of anomalies to the eastern equator and their growth after the strong westerly wind anomalies, with the seasonal migrations of the thermocline appears to explain the preference for winter that the events of type II display in fig. 3a.

The autumnal signature of events of type I, shown in fig. $3 \mathrm{a}$, can also be related to the sea- sonal displacements of the thermocline. In this case, the transit of subsurface anomalies to the east occurs in months -10 to -6 , and the strongest westerly wind anomalies and subsequent enhancement of temperature anomalies in the east in months -3 to -1 . The peak of Niño3 Index in autumn can be explained again by an association of these features with the climatological January-March and July-September movements of the thermocline.

\section{Classification of cold events according to their generation}

Using a criterion equivalent to the one above (now SST anomalies must fall under a negative threshold), 36 cold events are identified from the Niño3 Index, and a classification procedure identical to the one followed in the case of the warm events applied to them. Cold events appear to be generated along two different ways only. The statistical details of this classification are given in table II. It can be also appreciated there how the distance between the centers of each cluster exceeds the value of the average distance within a group plus one standard deviation.

For these cold events, the most important differences occur outside the equatorial strip. Figure 6 shows the most characteristic feature: negative $\mathrm{HC}$ anomalies 6 months before the peak of the event are in the West Pacific, south of the equator for events of type I (top row, left) and north of it for events of type II (bottom row, left). At this time, SST anomalies in the East Pacific are already negative in events of type I, while they are still positive in events of type II.

Table II. Cold events classification results. N1, D1, S1, N2, D2, S2, FV: same as in table I but for cold events.

\begin{tabular}{cccccccc}
\hline \hline Group & N1 & D1 & S1 & N2 & D2 & S2 & FV \\
\hline I & 13 & 0.32 & 0.15 & 23 & 0.75 & 0.19 & $39 \%$ \\
II & 12 & 0.44 & 0.15 & 24 & 0.71 & 0.22 & $38 \%$ \\
\hline
\end{tabular}



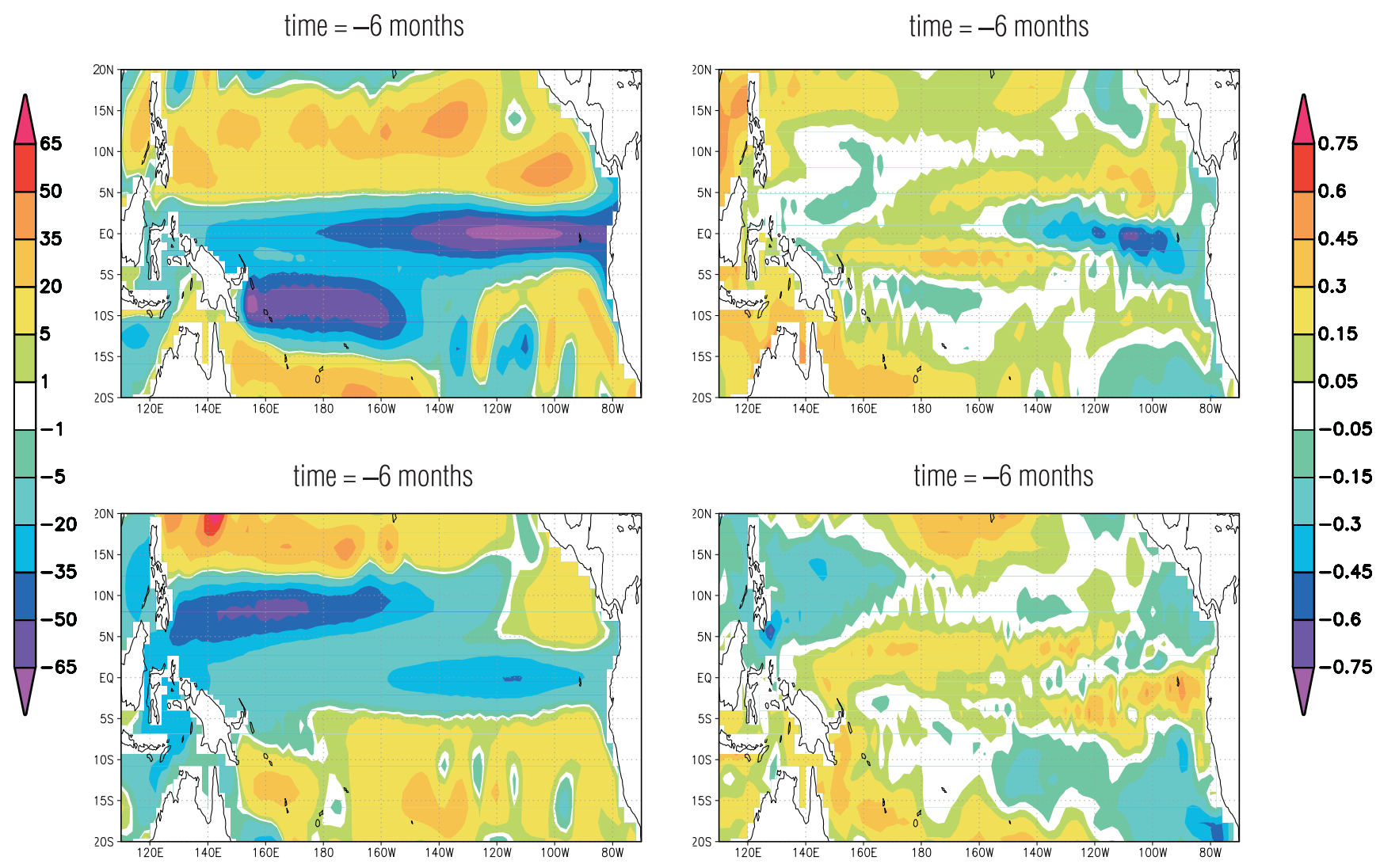

Fig. 6. Left: Heat Content $\left(10^{7} \mathrm{Jm}^{-2}\right)$ and right: $\mathrm{SST}\left({ }^{\circ} \mathrm{C}\right)$ anomalies 6 months in advance of a cold SST anomaly peak for events type I (top) and II (bottom).

\subsection{The cold events phase locking}

The seasonal signatures for the simulated and observed cold events are presented in fig. 7a and $7 \mathrm{~b}$, respectively. There seems to be a tight locking of cold events of type II to May-June, which appears to be related to the simulation's climatological shallowing of the thermocline in the eastern Pacific during the winter-spring months. The cold HC anomalies accumulated in the western part of the basin are not as strong as in events of type I, but the said seasonal feature would act to amplify them once they reach the eastern equator. Locking to the seasonal cycle is less strong in the case of events of type I, which do not seem to be so dependent on a seasonal amplifying mechanism. The presence of a seasonal effect is nevertheless suggested by the preferential occurrence of events in September. This tendency points, as in warm events of types I and II, to a connection between the eastbound transit of anomalies from the western margin with the seasonal rising of the equatorial thermocline in the Central Pacific in January-March.

\section{Interaction with extra-basin signals}

In our analysis of the tropical Atlantic simulated with the ECHAM4-OPYC3 (Cabos Narváez et al., 2002), a research into the connection of the different types of events with the ENSO signal, represented by the Niño3 Index, has proven very fruitful. We would like to follow the same line to investigate the influence of other climatic signals outside the tropical Pacific on ENSO variability. Possible candidates for these signals must be found in the tropics, to exclude the midlatitudes trend, present in the SINTEX simulation, from the analysis. 
(a)
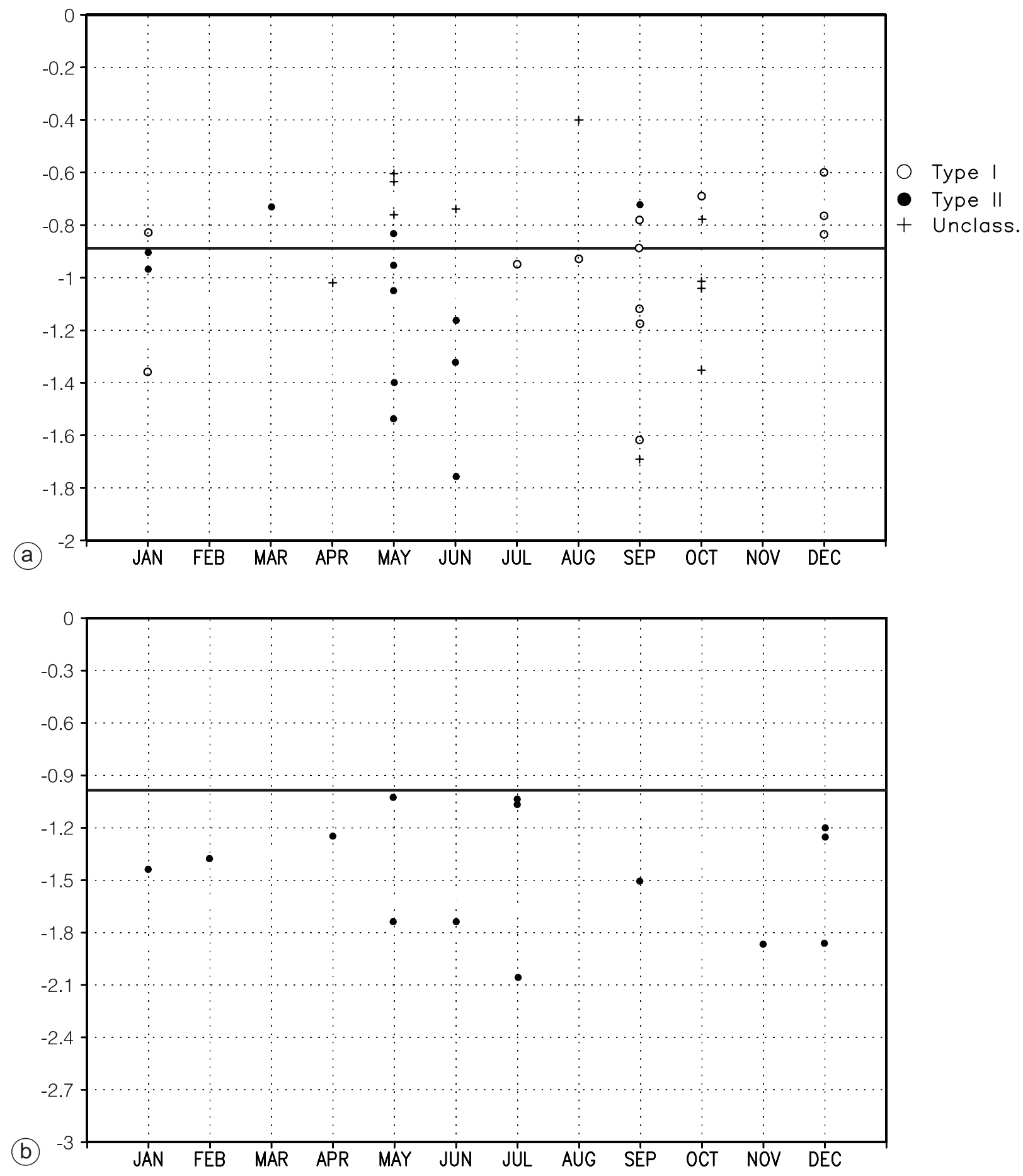

Fig. 7a,b. a) Seasonal distribution of cold SST anomaly peaks in the SINTEX simulation. The horizontal line marks the 1.5 standard deviation threshold for the Niño3 Index; b) as in (a), but for the observed SST anomalies (COADS + IGOSS, 1950-1999) peaks.

Figure 8a shows the connection between the Gulf of Guinea (or GG) Index, representing the tropical Atlantic variability, and the Niño3 Index. The three curves depicted correspond to the period of occurrence of different types of warm events. Juxtaposing chunks of 19 months, centered at the peak of each event, correlations with the GG lndex are computed. Maximum 
(a)
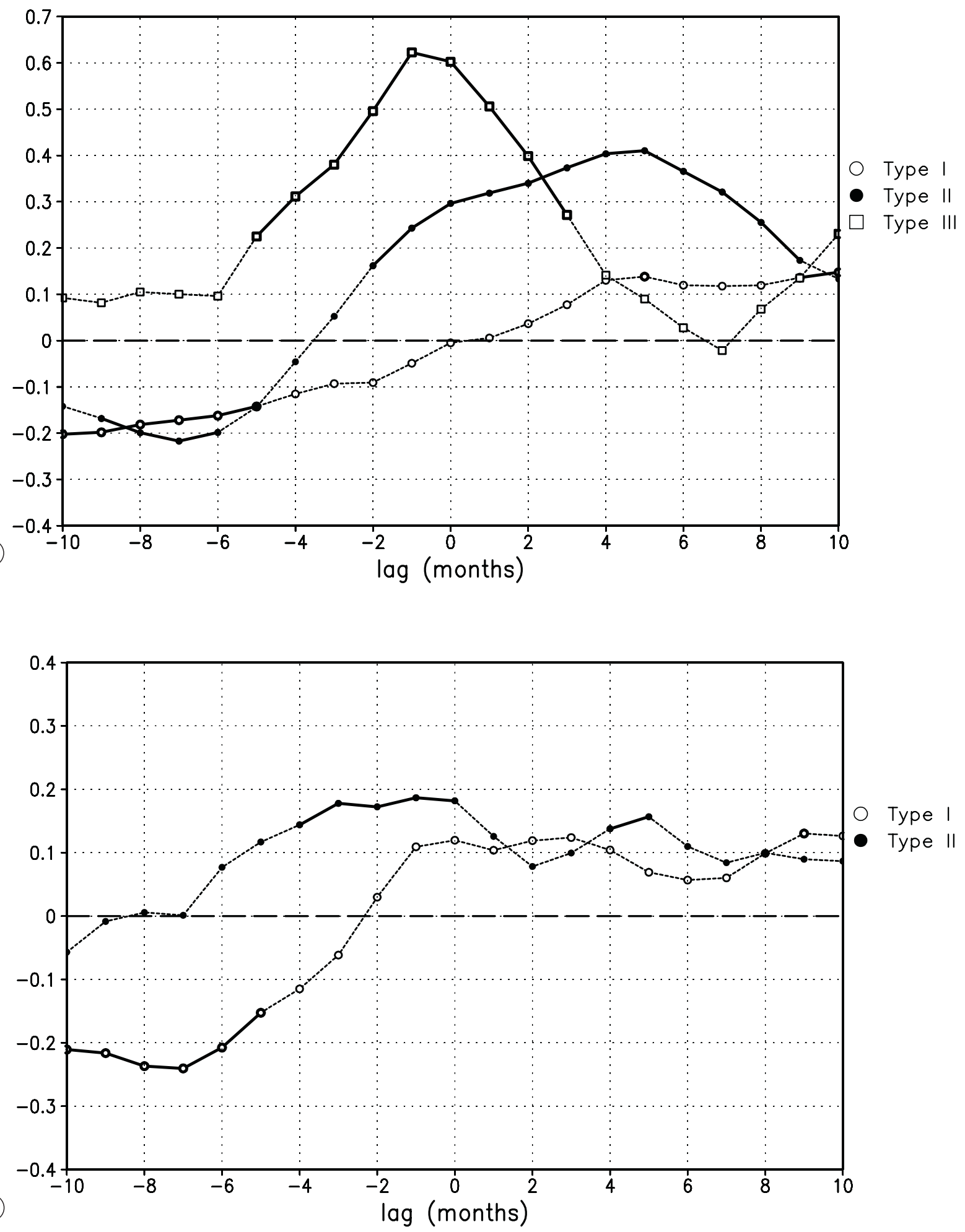

Fig. 8a,b. a) Lagged correlation between the SINTEX simulation GG and Niño3 Indices (GG leading at negative lags). Different time series have been formed by isolating the periods of occurrence of the distinct types of warm ENSO events. Solid thick lines correspond to correlation values significantly greater than zero at the 5\% level; b) as in (a), now for cold ENSO events. 
significant correlations with the GG Index are found for events of type III at lags between -3 months (GG leading) and +3 months (GG lagging). For events of type I (those that begin their generation earlier) the significant values of the correlation appear at greater negative lags, while for the type II events (the more intense ones) negative significants values are found at shorter negative lags. For these last, there are also significant positive values with the Gulf of Guinea Index lagging.

In the case of cold events, the type I only has a significant correlation (negative) when the GG Index precedes from 10 to 5 months. The correlation in the case of the type II events is positive and significant when the GG Index precedes from 4 up to 0 lags.

\section{Summary and conclusions}

A spectral analysis of the Niño3 Index of the SINTEX experiment reveals the presence of a 4 year peak, in addition to the dominant biennial timescale. This realistic feature is absent from the spectra of the other coupled simulation analyzed. A possible explanation for the absence of the four years peak in the ECHAM4-OPYC3 might lie in the different interactions between the interannual and seasonal variability. This last hypothesis is tested here by means of a higher order spectral analysis, which detects in the bicoherence of the ECHAM4-OPYC a strong interaction between the biennial variability and the annual and semiannual peaks. This interaction, not present in the SINTEX run could divert the energy from the interannual to the intraannual variability. This feature can be perhaps related to the flux correction applied to the ECHAM4-OPYC3, which, on the other hand, allows a satisfactory simulation of the midlatitudes and thereby of the tropical-midlatitudes interactions.

The similarities and differences among the warm ENSO events in the SINTEX simulation are investigated with help of a spatio-temporal cluster analysis. Warm events can be sorted into three classes. A common feature in the generation of two of these classes is the accumulation of $\mathrm{HC}$ anomalies in the west Pacific, north and south of the equator, well before the start of the events. The majority of the events belongs to these groups. But there is a minority in which the HC anomalies appear in the central equatorial Pacific.

The generation of the cold events present in the SINTEX run can be explained basically with only two classes. The most characteristic difference is the position, six months before the peak of the event, of the maxima of the negative $\mathrm{HC}$ anomalies. In the first type, it is south of the equator, north of it in the other.

The influence of the climatological cycle is evident in both warm and cold events and appears to be linked to the seasonal migrations of the equatorial thermocline. Thus, $\mathrm{HC}$ anomalies that generate warm events of types I and II are enhanced if they coincide with those displacements, leading to stronger amplitudes of the events. In a sense, these two classes of events could be regarded as two alternate ways of adjustment of ENSO to the annual cycle, which connects our results to ideas outlined in previous works with simple and hybrid models (Neelin et al., 2000).

The same mechanism of phase locking to the climatology seems to intervene in cold events, especially in those of type II, which show a particularly tight locking to the annual cycle, also related to seasonal changes in the thermocline position in the Eastern Pacific.

Lastly, the connection of the Pacific variability to other tropical signals has been examined bearing in mind the classification of both, warm and cold events. Values of the correlation between the Gulf of Guinea Index and the different types of warm events (fig. 7a) point to events of type III being preceded by tropical Atlantic warmings, while warm events of type II are sometimes preceded by tropical Atlantic cold events. The relationship in the case of the cold event types is not as strong as in the case of the warm events.

\section{Acknowledgements}

F. Álvarez wishes to acknowledge LODYC's hospitality during a short stay there. The authors are grateful to the anonymous reviewers for their useful comments. This work was supported by 
contract ENV4-CT98-0714 (SINTEX) from the European Union Environmental and Climate program.

\section{REFERENCES}

ACHUTA RAO, K., K.R. SPERBER and the CMIP MODELLING Group (2000): The El Niño-Southern Oscillation in coupled GCMs, PCMDI Report Series, 61, pp.46

Álvarez García, F.J., W.D. CABOS NARVÁEZ and M.J. ORTIZ BEVIÁ (2002): Different kinds of ENSO generation in a coupled GCM simulation, Climate Dyn. (in revision).

BACHER, A., J.M. OBERHUBER and E. ROECKNER (1998): ENSO dynamics and seasonal cycle in the tropical Pacific as simulated by the ECHAM4-OPYC3 coupled general circulation model, Climate Dyn., 14, 431-450.

Cabos Narváez, W.D., F.J. Álvarez García and M.J. ORTIZ BEVIÁ (2002): Generation of equatorial Atlantic warm and cold events in a coupled GCM simulation, Tellus A, 54, 426-438.

CHAO, Y. and S.G.H. PHILANDER (1993): On the structure of the southern oscillation, J. Climate, 6, 450-469.

ElgAR, S. and G. SEBERT (1989): Statistics of bicoherence and biphase, J. Geophys. Res., 94, 10,993-10,998.

GuAldi, S., A. NAVARRA, E. GuILYARDi and P. DELECluse (2002): Assessment of the tropical Indo-Pacific climate in the SINTEX CGCM, Ann. Geophysics, 46 (1), 1-26 (this volume).

LAtif, M., A. Sterl, E. MAier-Reimer and M. Junge (1993): Climate variability in a coupled GCM, part I: the tropical Pacific, J. Climate, 6, 5-21.

MAdeC, G., P. DeleCluse, M. IMBARD and C. LeVy (1998): OPA Version 8.1 ocean general circulation model reference manual, Tech. Rep., LODYC/IPSL Note 1.1.

NEELIN J.D., F.-F. JIN and H.-H. SYU (2000): Variations in ENSO phase locking, J. Climate, 13, 2570-2590.

RASMUSSON, E.M. and T.H. CARPENTER (1982): Variations in tropical sea surface temperature and surface wind fields associated with the southern oscillation/El Niño, Mon. Weather Rev., 110, 354-384.

ROECKNER, E., J.M. OBERHUBER, A. BACHER, M. CRISTOPH and I. KIRCHNER (1995): ENSO variability and atmospheric response in a global coupled oceanatmosphere GCM, Max Planck Institut für Meteorologie, Report n. 170, Hamburg.

Roeckner, E., K. Arpe, L. Bengtsson, M. CRistoph, L. Duemenil, M. Esch, M. GiorgetTA, U. Sclese and U. SCULZWEIDA (1996): The atmospheric general circulation model ECHAM4: model description and simulation of present day climate, Max Planck Institut für Meteorologie, Report n. 218, Hamburg.

TREnBerTh, K.E. (1997): The definition of El Niño, Bull. Amer. Meteorol. Soc., 78, 2771-2777.

WYRTKI, K. (1985): Water displacements in the Pacific and the genesis of El Niño cycles, J. Geophys. Res., 90, 7129-7132. 